\title{
Mid-term evaluation of the safety and efficacy of the iStent trabecular micro-bypass system combined with phacoemulsification
}

\author{
Milena Kozera ${ }^{1, A-D}$, Joanna Konopińska ${ }^{2, E}$, Marek Rękas ${ }^{1, A, F}$ \\ ${ }^{1}$ Department of Ophthalmology, Military Institute of Medicine, Warszawa, Poland \\ ${ }^{2}$ Department of Ophthalmology, Medical University of Białystok, Poland \\ A - research concept and design; $\mathrm{B}$ - collection and/or assembly of data; $\mathrm{C}$ - data analysis and interpretation; \\ $\mathrm{D}$ - writing the article; $\mathrm{E}$ - critical revision of the article; $\mathrm{F}$ - final approval of the article
}

Address for correspondence

Milena Kozera

E-mail:m.kozera@onet.eu

Funding sources

None declared

Conflict of interest

None declared

Received on July 16, 2020

Reviewed on August 6,2020

Accepted on 0ctober 29, 2020

Published online on January 30, 2021

Cite as

Kozera M, Konopińska J, Rẹkas M. Mid-term evaluation

of the safety and efficacy of the iStent trabecular

micro-bypass system combined with phacoemulsification.

Adv Clin Exp Med. 2021;30(1):49-54. doi:10.17219/acem/129576

DOI

10.17219/acem/129576

Copyright

Copyright by Author(s)

This is an article distributed under the terms of the

Creative Commons Attribution 3.0 Unported (CC BY 3.0)

(https://creativecommons.org/licenses/by/3.0/)

\begin{abstract}
Background. Micro-invasive glaucoma surgery (MIGS) and MIGS devices have been gaining increasing attention in recent years. One such device is the trabecular micro-bypass stent, or iStent ${ }^{\circledast}$ (Glaukos Corporation, Laguna Hills, USA).

Objectives. To evaluate the safety and efficacy of the minimally invasive ab interno surgical implantation of a trabecular bypass during cataract surgery in reducing intraocular pressure (IOP) in patients with mild and moderate open-angle glaucoma and cataracts.
\end{abstract}

Material and methods. The study was a prospective, uncontrolled, interventional case series (a prospective study of a case series), including 54 patients with a mean age of 72 years. All subjects underwent ab interno implantation of a single iStent together with cataract surgery. The corrected distance visual acuity (CDVA), IOP, anti-glaucoma medications, visual field, and number and type of complications were investigated after surgery. The patients were followed up at 1, 7, and 30 days, and 3, 6, 12, 24 and 36 months after the operation.

Results. The mean observation time was 20 months. At baseline, CDVA was 0.5 or better in $65 \%$ of the eyes; this improved to 0.5 or better in all eyes ( 0.8 or better in $79 \%$ ) at the end of the observation. The mean baseline $10 \mathrm{P}$ was $17.1 \mathrm{~mm} \mathrm{Hg}$, which fell to a mean of $15.1 \mathrm{~mm} \mathrm{Hg}$. The mean number of medicinal eye drops prescribed preoperatively was 1.7 , which decreased to 0.26 at the end of the observation.

Conclusions. Cataract surgery combined with iStent implantation seems to be an effective procedure in patients with mild to moderate open-angle glaucoma and cataracts. The insertion of 1 stent resulted in a significant decrease in IOP and a reduction in the number of topical anti-glaucoma medications needed. Based on the characteristics of the observed complications, iStent implantation can be considered a safe method.

Key words: open-angle primary glaucoma, micro-invasive glaucoma surgery, iStent ${ }^{\oplus}$ 


\section{Introduction}

Glaucoma is the second most common cause of blindness in the world and one of the major causes in Europe. It is a group of diseases that share a common feature: progressive damage to the optic nerve and related visual field defects. The main risk factor for the development of glaucoma neuropathy is elevated intraocular pressure (IOP). Reducing this pressure is still the only proven method of treating glaucoma. ${ }^{1}$

A common treatment of newly diagnosed mild to moderate open angle glaucoma (OAG) begins with local antihypertensive therapy, though laser trabeculoplasty is an alternative. The traditional approach to glaucoma treatment to date involves the use of surgical techniques as the ultimate therapeutic method. With the use of filtration procedures, which are the most common methods, obtaining good pressure control is associated with the risk of numerous intra- and postoperative complications. In addition, the use of anti-metabolites increases the risk of complications such as hypotension, leakage, filter bubble infection, and endophthalmitis. ${ }^{2}$

A better understanding of the pathophysiology of glaucoma, especially aqueous humor outflow pathways, has resulted in newer treatments. Starting from ab externo procedures, such as trabeculectomy or viscocanalostomy, through canoplasty, a group of ab interno treatments has been created, resulting in minimal trauma to the target tissue without affecting the anatomical structures and physiology of the eye, less invasiveness, good efficiency, and a high safety profile. ${ }^{3}$ A common term for these procedures is micro-invasive glaucoma surgery (MIGS). ${ }^{4}$ The MIGS uses different types of implants. One of the first stents to be placed in the Schlemm's canal was the iStent ${ }^{\circledR}$ microstent (Glaukos Corporation, Laguna Hills, USA). This is one of the smallest medical implants ever used in humans. Many prospective multicenter clinical trials have shown that the iStent ${ }^{\circledR}$ safely and effectively reduces IOP while reducing or eliminating the need for antihypertensive drugs. The data shows that the implantation of a single iStent in conjunction with a cataract surgery significantly reduces IOP for up to 5 years after surgery in patients with glaucoma and cataracts. ${ }^{5-11}$

To investigate the potential usefulness of the microstent as a therapy in patients with early and intermediate glaucoma and cataracts, we conducted a prospective case series study assessing the efficacy and safety of a single iStent implant in surgery combined with cataract phacoemulsification.

\section{Material and methods}

\section{Patients and study protocol}

A prospective single-center study was conducted inwhich patients with primary open-angle glaucoma and cataracts were enrolled. The study protocol was in line with the principles of the Declaration of Helsinki and was approved by the Bioethics Committee at the Military Medical Institute (Warszawa, Poland). Prior to recruitment, legally binding, informed consent was obtained from each patient for Glaukos iStent implantation and cataract surgery.

The inclusion criteria were an IOP $\leq 31 \mathrm{~mm} \mathrm{Hg}$; the use of up to 4 antihypertensive drugs; open angle confirmed with gonioscopy; and mild (mean deviation (MD) from 0 to -6.0 $\mathrm{dB}$ ) or moderate (MD from -6.0 to $-12 \mathrm{~dB}$ ) defects in the field of view confirmed using the ZEISS Humphrey Field Analyzer (Carl Zeiss AG, Jena, Germany) with the SITA Standard 24-2 program (Ophthalmological Clinic Military Institute of Medicine, Warszawa, Poland). Other inclusion criteria included a pre-operative best-corrected visual acuity (BCVA) of 20/200 or better, and the willingness to attend follow-up appointments for the duration of the study, i.e., 12 months.

The key exclusion criteria were forms of glaucoma other than primary open-angle glaucoma and pseudoexfoliative glaucoma; evidence of serious eye diseases other than glaucoma and senile cataracts, such as proliferative diabetic retinopathy, corneal diseases (e.g., Fuchs dystrophy), age-related macular degeneration - the dry or exudative form; a narrow angle of filtration; previous anti-glaucoma treatment (trabeculoplasty, trabeculectomy, filtration, cyclocoablation, etc.); previous surgery not caused by glaucoma or cataracts, except for ophthalmic procedures; glaucoma defects in the field of view with an $\mathrm{MD}<-12 \mathrm{~dB}$, as confirmed with a Humphrey field analyzer; and the use of more than 4 anti-glaucoma drugs.

\section{iStent and surgical technique}

An iStent ${ }^{\circledR}$ is a one-piece, L-shaped, heparin-coated titanium implant smaller than $1 \mathrm{~mm}$ and approx. $0.3 \mathrm{~mm}$ high. The iStent microfistula is inserted through a small incision in the transparent part of the cornea. It is used to drain the aqueous humor directly into the Schlemm's canal. The stent is introduced ab interno into the Schlemm's canal using a spring mechanism with a diameter of $27 \mathrm{G}$. One or more implants can be inserted into the corner on the nasal side. When the fistula is in place, a small blood reflux may occur, usually self-limiting in nature. The iStent implantation does not bypass the physiological outflow pathway, but supports it in the hope of achieving an acceptable IOP level and reducing or eliminating the use of anti-glaucoma drugs.

The surgical procedure was performed under local anesthesia with $2 \%$ xylocaine. After cataract removal and the creation of an artificial intraocular posterior chamber with phacoemulsification (facochop), the implantation of iStent began. The right stent was used in the right eyes and the left stent was used in the left eyes. The patient's eyes were positioned nasally, and the microscope was turned $30^{\circ}$ from the surgeon. A viscoelastic agent was administered into the anterior chamber for visibility. Through the existing temple opening in the corneal limbus, the iStent was introduced into the trabecular mesh at an angle of $20^{\circ}$ and 
moved deeper. A spring injector was used for stent implantation, while a Swan-Jacobs gonioscope was used to visualize the angle of filtration. The "impact" of the stent on the tip of the injector from back to front confirmed the correct placement of the microimplant. After the applicator was withdrawn, the remaining viscoelastic agent was removed by irrigation and aspiration. The anterior chamber was sealed with saline solution and intraocular pressure was controlled to complete the surgery. All procedures were performed by the same surgeon (MR) in the Department of Ophthalmology of the Military Institute of Medicine.

\section{Postoperative management}

The patients used antibiotic and anti-inflammatory eye drops for 4 weeks. Postoperative check-ups took place on day 1, 7 and 30, and after 3, 6, 12, 24, and 36 months. At each examination, the BCVA was assessed with Snellen chart, IOP was measured with a Goldmann applanation tonometer, and the anterior segment and the fundus was examined in the slit lamp. The gonioscopic angle was also assessed (except for the $1^{\text {st }}$ day after surgery). At the 12 and 24-month follow-up, the field of view was taken using a Humphrey apparatus with the SITA Standard 24-2 program. An important parameter evaluated in the study was the number of anti-glaucoma drugs used. Additional information collected throughout the study was related to treatment and/or study-related adverse effects.

\section{Statistical analysis}

The effectiveness of the procedures used in the study was analyzed on the basis of all available data from IOP measurements and the number of local antihypertensive drugs. A total IOP reduction to $\leq 15 \mathrm{~mm} \mathrm{Hg}$ was considered independent of treatment, while a reduction in IOP to $\leq 18 \mathrm{~mm} \mathrm{Hg}$ indicated a "partial success."

For the continuous variables, 2 mock tests were used to assess differences between the groups. Fisher's exact test was used to compare categorical results between the groups. To assess the effect of time and intervention on the measured parameters, linear mixed models with random constant expression were built for the patient. Model coefficients are reported with a 95\% confidence interval (95\% CI) and p-value; the level of the latter of 0.05 was considered statistically significant. All analyses were performed using the Statistical Analysis System (SAS) statistical software package v. 9.1.3. (SAS Institute Inc., Cary, USA).

\section{Results}

\section{Demographics}

The study involved 54 eyes of 52 patients. The study group included 38 women and 14 men with a mean age
Table 1. Demographics and preoperative variables

\begin{tabular}{|l|c|}
\hline \multicolumn{1}{|c|}{ Variable } & Value \\
\hline Total & 54 eyes of 52 patients \\
\hline Age [years] & $72(8)$ \\
\hline Gender (F/M) & $38 / 14$ \\
\hline Race - Caucasian & $100 \%(n=54)$ \\
\hline $\begin{array}{l}\text { Type of glaucoma } \\
\text { primary open-angle glaucoma } \\
\text { pseudoexfoliative glaucoma }\end{array}$ & $94 \%(n=51)$ \\
\hline Preoperative visual field (MD) [dB], mean (SD) & $6 \%(n=3)$ \\
\hline Preoperative visual field (PSD) [dB], mean (SD) & $-4.8(3.9)$ \\
\hline Preoperative S/D ratio, mean (SD) & $3.7(2.5)$ \\
\hline Preoperative medicated IOP [mm Hg], mean (SD) & $0.6(0.2)$ \\
\hline $\begin{array}{l}\text { Preoperative anti-glaucoma medications, } \\
\text { mean (SD) }\end{array}$ & $17.1(3.5)$ \\
\hline Preoperative BCVA & $1.7(0.9)$ \\
\hline $\begin{array}{l}20 / 25 \text { or better } \\
20 / 40 \text { or better }\end{array}$ & $17 \%(n=9)$ \\
\hline
\end{tabular}

BCVA - best-corrected visual acuity; MD - mean deviation; SD - standard deviation.

of 72 years (standard deviation $(\mathrm{SD})=8$ years). Most of the eyes $(94 \% ; \mathrm{n}=51)$ had primary open-angle glaucoma; $6 \%(n=3)$ had pseudoexfoliative glaucoma. Two patients had both eyes enrolled in the study. The mean follow-up period was 20 months $(S D=10)$. A total of 44 eyes were observed for 12 months, observation of 32 eyes was completed within 24 months, and observation of 10 eyes was extended to 36 months. Demographic data and descriptive characteristics are presented in Table 1.

\section{Intraocular pressure and medications}

The baseline mean intraocular pressure was $17.1 \pm 3.5$ $\mathrm{mm} \mathrm{Hg}$. The last study showed a decrease in IOP to mean values of $15.7 \pm 2.2 \mathrm{~mm} \mathrm{Hg}$; similar results were found after 12 months (Fig. 1).

A reduction in IOP to a value $\leq 15 \mathrm{~mm} \mathrm{Hg}$ occurred in $43 \%$ of the eyes (compared to $26 \%$ before surgery) and $\leq 18 \mathrm{~mm} \mathrm{Hg}$ in $94 \%$ (compared with $63 \%$ before surgery; Fig. 2).

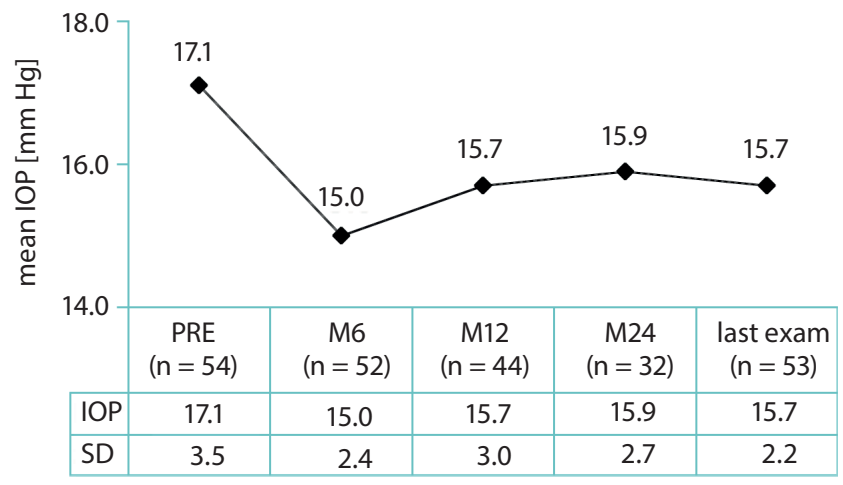

Fig. 1. Mean IOP at time intervals 


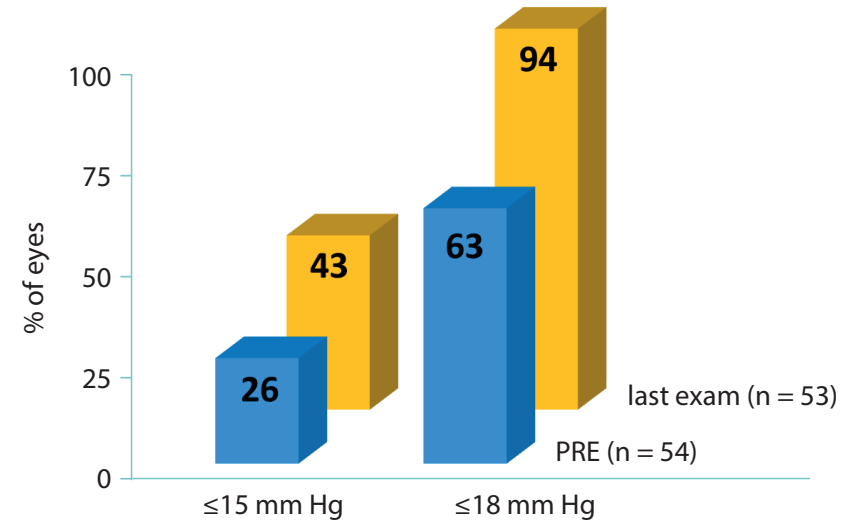

Fig. 2. Proportional analyses of IOP
There were no significant complications after cataract phacoemulsification surgery with iStent implantation. One patient had a subconjunctival hemorrhage on the $1^{\text {st }}$ day after surgery, in 5 eyes there were erythrocytes in the anterior chamber, and in 1 eye a corneal edema associated with an increase in IOP was revealed. All symptoms resolved completely within 7 days. One patient developed viral keratitis 1 week after surgery. Topical treatment was applied and the condition healed during the $1^{\text {st }}$ month. The final visual acuity in this patient was $20 / 20$. Table 2 presents the postoperative complications. Most complications occurred in the postoperative period and did not differ significantly from those after phacoemulsification of cataracts alone.

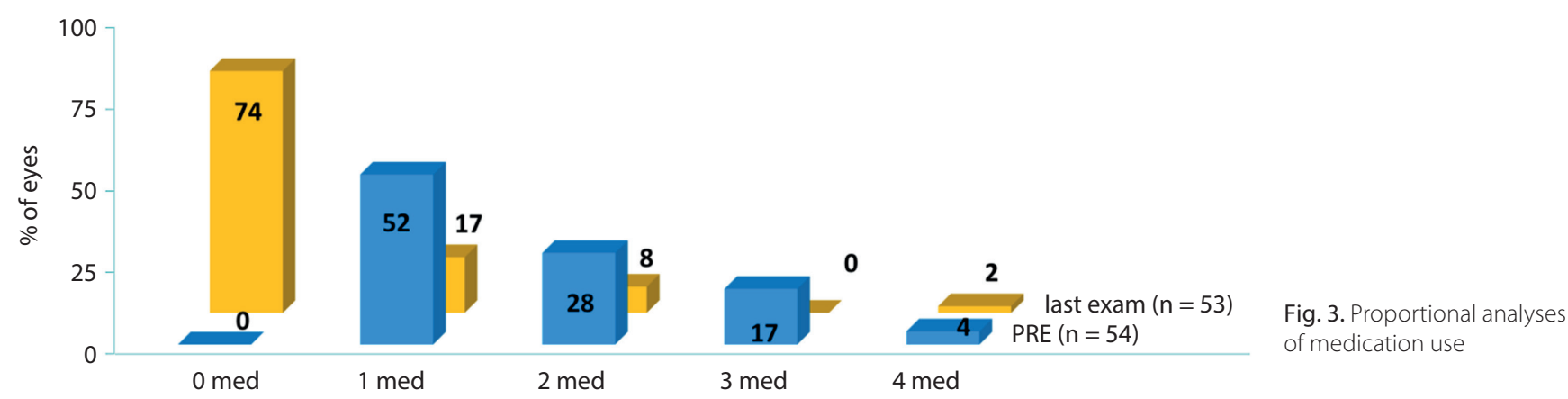

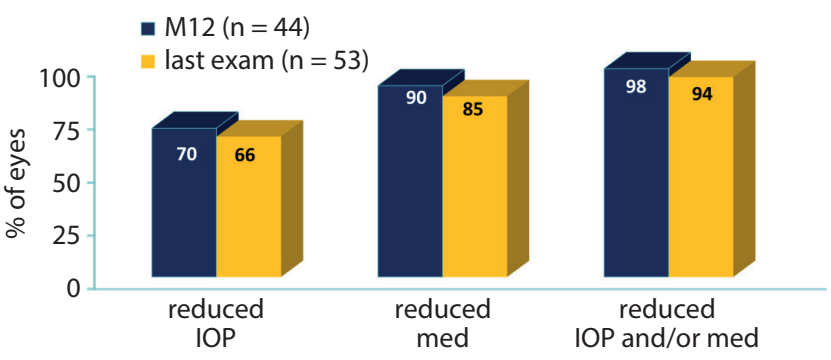

Fig. 4. IOP and medication reduction

The average number of drugs used before surgery was $1.7 \pm 0.9$. Drug consumption decreased significantly. In a recent study, $74 \%$ of patients were able to eliminate topical medications, compared to $49 \%$ of patients on 2 or more drugs in their preoperative examinations (Fig. 3). After 12 months, 70\% of patients had achieved a reduction in IOP, 90\% had reduced the number of drugs, and $98 \%$ had reduced IOP and/or drug use (Fig. 4). At the end of the follow-up period, $66 \%$ of patients had lower IOP than before surgery, $85 \%$ had reduced their use of antihypertensive eye drops, and $94 \%$ of patients had achieved a reduction in IOP and/or a reduction in drug use.

\section{Safety}

In no case did the visual acuity deteriorate. In the whole study group at the end of observations, BCVA was 20/40; in $79 \%$ of cases, BCVA had reached 20/25 (Fig. 5).

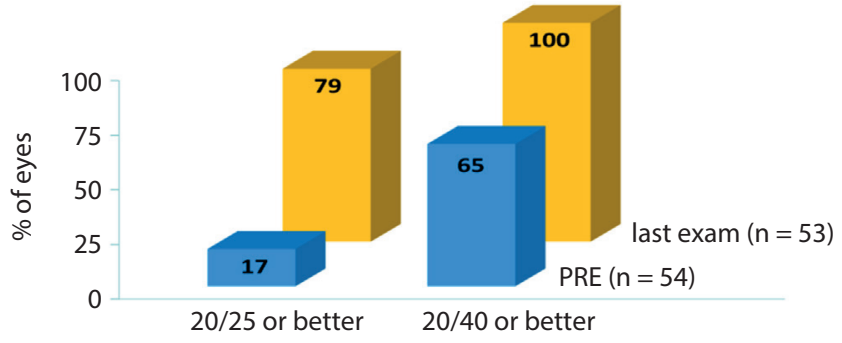

Fig. 5. Preoperative and last reported BCVA

Table 2. Postoperative complications

\begin{tabular}{|l|c|}
\multicolumn{1}{|c|}{ Complications } & $\mathrm{n}(\%)$ \\
\hline Conjunctival hemorrhage & $1(1.8)$ \\
\hline Temporary increase of IOP & $1(1.8)$ \\
\hline Single erythrocytes in anterior chamber & $5(9.2)$ \\
\hline Corneal erosion & $4(7.4)$ \\
\hline Viral keratitis & $1(1.8)$ \\
\hline
\end{tabular}

\section{Discussion}

Micro-invasive glaucoma surgery covers 3 anatomical areas: Schlemm's canal, the suprachoroidal and subconjunctival spaces. ${ }^{4}$ Because $80 \%$ of the aqueous humor drains through the conventional route, methods have been sought for decades to improve the outflow of aqueous humor using trabecular formulations. Ab externo 
trabeculotomy was one such method, ${ }^{12,13}$ used for the first time in adults by Tanihara et al. ${ }^{14}$ It was discovered that greater efficiency can be obtained by introducing a nonabsorbable thread with prolenol into the Schlemm's canal, stretching its walls. The use of microimplants in MIGS implanted into the Schlemm's canal was another step in the quest to develop an effective, less invasive method. Performing surgery with an ab interno approach does not cause damage to eye tissues such as the conjunctiva and the sclera.

Samuelson et al. described the use of a single iStent in a surgery combined with phacoemulsification of cataracts. ${ }^{11}$ In our report, the patients who had undergone stent implantation had a reduction in IOP of $66 \%$ and the average number of antihypertensive drugs decreased. The results of our study are in line with previous reports on the effectiveness of using iStents to reduce IOP and topical drug use, as well as those regarding the high safety profile of the implant. ${ }^{5-11}$ The results show that more than $90 \%$ of patients experienced a reduction in IOP, a reduction in the number of drugs used or a reduction in both.

The Early Manifest Glaucoma Trial reported that the risk of glaucoma progression is reduced by $10 \%$ with every millimeter of mercury reduced from baseline IOP. ${ }^{15}$ Slowing or arresting disease progression is the goal of glaucoma treatment, and as stated in the Early Manifest Glaucoma Trial analysis, even small decreases in IOP are clinically relevant. In our study, the average decrease in IOP was $2 \mathrm{~mm} \mathrm{Hg} 6$ months after surgery, with a sustained reduction for 12 months after surgery. Moreover, in about $50 \%$ of the eyes $(n=32)$, the follow-up time was extended to 2 years, and the IOP was $<16 \mathrm{~mm} \mathrm{Hg}$. Because only 3 patients had pseudoexfoliative glaucoma, we did not further investigate the results by type of glaucoma. This could constitute a topic for further research. In addition, followup of all patients is being continued for 3 years to allow future coherent cohort analysis with long-term follow-up.

Our study has several limitations. The study was an open and non-randomized case series study. Baseline IOP without wash-out was analyzed. In their work, Ferguson et al. ${ }^{16}$ noticed a correlation between preoperative IOP and the degree of IOP reduction observed 2 years after surgery. Patients with higher preoperative IOP achieved a significantly greater average reduction in IOP; in eyes with a preoperative IOP of $26 \mathrm{~mm} \mathrm{Hg}$ or more, an average reduction of $11.28 \mathrm{~mm} \mathrm{Hg}$ was obtained. Our study was a prospective study showing the results in reducing both IOP and the use of anti-glaucoma drugs at each time point. In addition, all the procedures were performed by 1 surgeon (MR) in 1 center.

The use of the iStent reduced the average number of drugs needed to control IOP in patients with open-angle glaucoma. Postoperatively, the average decrease in local anti-glaucoma drugs within the first 2 years after surgery was 1.3 fewer drugs; $74 \%$ eliminated the need for antihypertensive agents completely. These data are consistent with the results of other studies, in which the number of antiglaucoma eye drops used after cataract surgery with iStent implantation was monitored. ${ }^{4,11,17}$ Non-compliance with medical recommendations and a lack of regularity in taking anti-glaucoma drugs is still a serious problem, which may be responsible for nearly $10 \%$ of vision loss. ${ }^{18}$ Therefore, the goal of surgical treatment is not only a reduction in IOP, but also the elimination of the need for topical drugs that, when used chronically, have an adverse effect on the surface of the eyeball and reduce the effectiveness of filtration operations.

Micro-invasive procedures using ministents have a high safety profile. ${ }^{5-11}$ There are no complications like those following fistula follicle operations: hypotension, excessive filtration, infection, or early follicular overgrowth. Early undesirable symptoms after cataract surgery with iStent implantation are similar to those after cataract surgery alone. In our study, symptoms such as corneal erosion, conjunctival hemorrhage and erythrocytes in the anterior chamber disappeared within the first week. In addition, the rehabilitation time was significantly shorter compared to filtration procedures and there was no need for additional procedures such as administering subconjunctival metabolites, suture lysis or needling. The surface of the eyeball was not affected during the procedure, thanks to which there is still an unlimited possibility to conduct more invasive anti-glaucoma procedures.

\section{Conclusions}

The results of our study of cataract surgery combined with single iStent implantation in patients with primary open-angle glaucoma or pseudoexfoliative glaucoma suggest that this is an effective procedure. The implantation of the ministent caused a permanent decrease in intraocular pressure as well as in the number of topical antiglaucoma drugs. Based on the types of complications observed within the first 3 years, this surgical technique can be considered a safe method.

\section{ORCID iDs}

Milena Kozera (1) https://orcid.org/0000-0001-7156-3036 Joanna Konopińska (1) https://orcid.org/0000-0002-9088-6938 Marek Rękas (1) https://orcid.org/0000-0003-0429-6649

\section{References}

1. Schmidt W, Kastner C, Sternberg K, et al. New concepts for glaucoma implants: Controlled aqueous humor drainage, encapsulation prevention and local drug delivery. Curr Pharm Biotechnol. 2013;14(1):98-111.

2. Soltau JB, Rothmann RF, Budenzo DL, et al. Risk factors for glaucoma filtering bleb infections. Arch Ophthalmol. 2000;118(3):338-342.

3. SooHoo JR, Seibold LK, Radcliffe NM, Kahook MY. Minimally invasive glaucoma surgery: Current implants and future innovations. Can J Ophthalmol. 2014;49(6):528-533.

4. Saheb H, Ahmed II. Micro-invasive glaucoma surgery: Current perspectives and future directions. Curr Opin Ophthalmol. 2012;23(2): 96-104. 
5. Neuhann TH. Trabecular micro-bypass stent implantation during small-incision cataract surgery for open-angle glaucoma or ocular hypertension: Long-term results. J Cataract Refract Surg. 2015;41(12): 2664-2671.

6. Fea AM, Consolandi G, Zola M, et al. Micro-bypass implantation for primary open-angle glaucoma combined with phacoemulsification: 4-year follow-up. J Ophthalmol. 2015;2015:795357.

7. Arriola-Villalobos P, Martinez-de-la-Casa J, Diaz-Valle D, FernándezPérez C, García-Sánchez J, García-Feijoó J. Combined iStent trabecular micro-bypass stent implantation and phacoemulsification for coexistent open-angle glaucoma and cataract: A long-term study. Br J Ophthalmol. 2012;96(5):645-649.

8. Belovay GW, Naqi A, Chan BJ, Rateb M, Ahmed IKK. Using multiple trabecular micro-bypass stents in cataract patients to treat openangle glaucoma. J Cataract Refract Surg. 2012;38(11):1911-1917.

9. Craven ER, Katz LJ, Wells JM, Giamporcaro JE; iStent Study Group. Cataract surgery with trabecular micro-bypass stent implantation in patients with mild-to-moderate open-angle glaucoma and cataract: Two-year follow-up. J Cataract Refract Surg. 2012;38(8):1339-1345.

10. Fea AM. Phacoemulsification versus phacoemulsification with micro-bypass stent implantation in primary open-angle glaucoma: Randomized double-masked clinical trial. J Cataract Refract Surg. 2010;36(3):407-412.

11. Samuelson TW, Katz LJ, Wells JM, Duh YJ, Giamporcaro JE; US iStent Study Group. Randomized evaluation of the trabecular micro-bypass stent with phacoemulsification in patients with glaucoma and cataract. Ophthalmology. 2011;118(3):459-467.
12. Burian HM. A case of Marfan's syndrome with bilateral glaucoma. With description of a new type of operation for developmental glaucoma (trabeculotomy ab externo). Am J Ophthalmol. 1960;50(6): 1187-1192.

13. Smith R. A new technique for opening the canal of Schlemm: Preliminary report. Br J Ophthalmol. 1960;44(6):370-373.

14. Tanihara $H$, Negi A, Akimoto $M$, et al. Surgical effects of trabeculotomy ab externo on adult eyes with primary open angle glaucoma and pseudoexfoliation syndrome. Arch Ophthalmol. 1993;111(12): 1653-1661.

15. Heijl A, Leske MC, Bengtsson B, Hyman L, Bengtsson B, Hussein M; Early Manifest Glaucoma Trial Group. Reduction of intraocular pressure and glaucoma progression: Results from the early manifest glaucoma trial. Arch Ophthalmol. 2002;120(10):1268-1279.

16. Ferguson TJ, Berdahl JP, Schweitzer JA, Sudhagoni RG. Clinical evaluation of a trabecular micro-bypass stent with phacoemulsification in patients with open-angle glaucoma and cataract. Clin Ophthalmol. 2016;10:1767-1773.

17. Spiegel D, Wetzel W, Neuhann T, et al. Coexistent primary open-angle glaucoma and cataract: Interim analysis of a trabecular micro-bypass stent and concurrent cataract surgery. Eur J Ophthalmol. 2009;19(3): 393-399.

18. European Glaucoma Society. Terminology and Guidelines for Glaucoma. $4^{\text {th }}$ ed. Savona, Italy: Dogma; 2014:1-191. 